\title{
Collisional excitation of water in warm astrophysical media
}

\section{Rate coefficients for rovibrationally excited states ${ }^{\star}$}

\author{
A. Faure ${ }^{1}$ and E. Josselin ${ }^{2}$ \\ 1 Laboratoire d'Astrophysique de Grenoble (LAOG), Université Joseph-Fourier, UMR 5571 CNRS, BP 53, \\ 38041 Grenoble Cedex 09, France \\ e-mail: afaure@obs.ujf-grenoble.fr \\ 2 Groupe de Recherche en Astronomie et Astrophysique du Languedoc (GRAAL), Université Montpellier II, UMR 5024 CNRS, \\ 34095 Montpellier Cedex 05, France
}

Received 30 July 2008 / Accepted 25 September 2008

\section{ABSTRACT}

\begin{abstract}
Context. The interpretation of water line emission from infrared and submillimetre observations requires a detailed knowledge of collisional rate coefficients over a wide range of levels and temperatures.

Aims. We attempt to determine rotational and rovibrational rate coefficients for $\mathrm{H}_{2} \mathrm{O}$ colliding with both $\mathrm{H}_{2}$ and electrons in warm, molecular gas.

Methods. Pure rotational rates are derived by extrapolating published data using a new method partly based on the information (phase space) theory of Levine and co-workers. Ro-vibrational rates are obtained using vibrational relaxation data available in the literature and by assuming a complete decoupling of rotation and vibration.

Results. Rate coefficients were obtained for the lowest 824 ro-vibrational levels of $\mathrm{H}_{2} \mathrm{O}$ in the temperature range $200-5000 \mathrm{~K}$. Our data is expected to be accurate to within a factor of $\sim 5$ for the highest rates $\left(\gtrsim 10^{-11} \mathrm{~cm}^{3} \mathrm{~s}^{-1}\right)$. Smaller rates, including the rovibrational ones, should be generally accurate to within an order of magnitude. As a first application of this data, we show that vibrationally excited water emission observed in evolved stars is expected to be at least partly excited by means of collisions.
\end{abstract}

Key words. molecular data - molecular processes - ISM: molecules

\section{Introduction}

Water molecules are found in a large variety of astronomical environments, ranging from interstellar molecular clouds to stellar photospheres, circumstellar envelopes, and comets. In these media, they represent a major reservoir of oxygen. Furthermore, water emission may dominate the cooling of the warm part (a few $100 \mathrm{~K}$ ) of these regions (Neufeld \& Kaufman 1993). The water molecule is also one of the main sources of radio (from the submillimetre to the centimetre domain) maser emission, which has the potential to enable detailed studies of physical conditions, source dynamics, and magnetic fields (Humphreys 2007). A quantitative modelling of the excitation and line formation processes is therefore essential to understand the nature of these media. Besides the approximations introduced into radiative transfer calculations, such analyses are hampered by the lack of reliable collisional rate coefficients, especially for highlying rotational and vibrational levels.

Despite progress in both laboratory and theoretical studies of $\mathrm{H}_{2} \mathrm{O}$ collisional excitation (see Barnes et al. 2004; Faure et al. 2007 , and references therein), there is still a crucial lack of stateto-state rate coefficients for water in vibrationally excited states. Current rotational data (Green et al. 1993 for $\mathrm{H}_{2} \mathrm{O}-\mathrm{He}$; Faure et al. 2004 for $\mathrm{H}_{2} \mathrm{O}$-electron; Dubernet et al. 2006; and Faure et al. 2007 for $\mathrm{H}_{2} \mathrm{O}-\mathrm{H}_{2}$ ) is also restricted to levels of energy below the first excited vibrational state, which lies at $1594.7 \mathrm{~cm}^{-1}$ above the ground state. The main difficulty in computing rate coefficients for higher levels relates to the fact that quantum

* Tables A.1-A.4 are only available in electronic form at the CDS via anonymous ftp to cdsarc.u-strasbg.fr (130.79.128.5) or via http://cdsweb.u-strasbg.fr/cgi-bin/qcat?J/A+A/492/257 scattering calculations become computationally prohibitive as soon as the number of coupled channels to be included in the (time independent) Schrödinger equation exceeds a few thousands. This imposes severe limitations especially when the projectile has internal degrees of freedom such as $\mathrm{H}_{2}$. The quantum wave packet approach (see e.g. Otto et al. 2008) could provide an efficient alternative to fully coupled channel calculations but to our knowledge it has yet to be developed for polyatomic targets. There is currently therefore no quantum calculations for $\mathrm{H}_{2} \mathrm{O}-\mathrm{H}_{2}$ that include vibrational motion and/or rotationally "hot" molecules $\left(E_{\text {up }} \gtrsim 2000 \mathrm{~K}\right)$. At the quasi-classical level, hot molecules can be handled but other limitations occur, such as the inability of classical mechanics to estimate low probability processes (see Faure et al. 2007, and references therein). As a result, to extend the collisional data of water to kinetic temperatures at which it becomes vibrationally excited ( $T \gtrsim 2000 \mathrm{~K}$ ), a new approach is necessary and needs to be developed. This is the objective of the present article.

The method of computation of collisional rate coefficients, and its application to $\mathrm{H}_{2} \mathrm{O}-\mathrm{H}_{2}$ and $\mathrm{H}_{2} \mathrm{O}$-electron rotational and rovibrational excitation is exposed in Sect. 2. First astrophysical implications, including cooling rates and critical densities, are discussed in Sect. 3.

\section{Collisional rate coefficients}

\subsection{Rotational rates}

\subsection{1. $\mathrm{H}_{2} \mathrm{O}-\mathrm{H}_{2}$}

Faure et al. (2007) computed quasi-classical rate coefficients for rotational de-excitation in the lowest 45 para and 45 ortho levels of $\mathrm{H}_{2} \mathrm{O}$ colliding with both para- and ortho- $\mathrm{H}_{2}$ in the 
temperature range $20-2000 \mathrm{~K}$. This corresponds to a total of 990 para and 990 ortho $\mathrm{H}_{2} \mathrm{O}$ downward rates for each temperature. We note that in these calculations para- and ortho- $\mathrm{H}_{2}$ were assumed to be (separately) thermalized at the kinetic temperature. The objective of this section is to estimate the rotational rates for thousands of transitions involving states above the lowest 45 .

Our analysis is inspired by the information theory approach developed by Levine and co-workers (see the review by Levine 1978). This approach was originally introduced as a procedure to compact and correlate the vast amounts of data, both theoretical and experimental, on molecular collisions. For the rotational excitation of a linear rotor by a structureless particle, the following expression for the rate coefficient was suggested by Procaccia \& Levine (1976):

$k\left(J \rightarrow J^{\prime} ; T\right)=A(T) k^{0}\left(J \rightarrow J^{\prime} ; T\right) \exp \left[-\theta_{R}(T)\left|\Delta E_{J J^{\prime}}\right| / k_{B} T\right]$.

In the above expression, $J$ is the rotor angula momentum, $k^{0}\left(J \rightarrow J^{\prime} ; T\right)$ is a "prior" rate, simply proportional to the phase space available for the final state, $A(T)$ is a temperaturedependent normalization factor, $\theta_{R}$ is an adiabaticity parameter, and $\Delta E_{J J^{\prime}}=\left(E_{J^{\prime}}-E_{J}\right)$ is the rotational energy transfer or energy gap. The deviation of the actual (measured or computed) rate, $k$, from its prior value, $k^{0}$, is called the surprisal. A number of studies demonstrated that the accuracy of the surprisal analysis is variable and that different systems show a variety of scatter in the surprisal plots (see in particular Green 1979). It was generally recognized, however, that it accounts properly for the underlying qualitative trends.

Following Eq. (1), the expression below can be assumed for the rotational rates of water:

$k(i \rightarrow j ; T)=g_{j} A(T) \exp \left[-\theta_{R}(T)\left|\Delta E_{i j}\right| / k_{B} T\right]$,

where $\{i, j\}$ specify the initial and final $\mathrm{H}_{2} \mathrm{O}$ rotational quantum numbers (e.g. $J_{K_{a}, K_{c}}$ and $J_{K_{a}^{\prime}, K_{c}^{\prime}}^{\prime}$ respectively and $g_{j}$ is the degeneracy in the $j$ level, i.e. $\left(2 J^{\prime}+1\right)$. To assess the reliability of Eq. (2), a set of rates of Faure et al. (2007) at $2000 \mathrm{~K}$ is plotted, in Fig. 1, as a function of the energy gap. Despite the large scatter of points, the correlation between the rates and the energy gap is significant with a correlation coefficient equal to 0.66 . More reliable or similar results were obtained for other temperatures. The slope $\theta_{R}(T)$ was found to vary between 0.1 and 4.4 over the temperature range $20-2000 \mathrm{~K}$, in agreement with the observations of Procaccia \& Levine (1976) for heteronuclear diatomics. $\theta_{R}(T) / k_{B} T$ was also found to tend to a constant value at $2000 \mathrm{~K}$, reflecting the fact that rates converge to a simple $T^{1 / 2}$ temperature dependence at high temperature (see also Procaccia \& Levine 1976).

The scatter of data points in Fig. 1 reflects a complicated competition between different dynamical effects, including the energy gap but also specific dynamical constraints. In particular, although no rigorous selection rules exist for inelastic collisions, propensity rules are generally observed. For water- $\mathrm{H}_{2}$ collisions, the "preferred" rotational transitions can be defined to be those for which (Faure et al. 2007):

$\Delta J=0, \pm 1, \pm 2 ; \quad \Delta K_{a}=0, \pm 1, \pm 2 ; \quad \Delta K_{c}=0, \pm 1, \pm 2$.

The above rules are of course not accounted for by the information theory approach.

Our analysis of the data of Faure et al. (2007) demonstrated that for a given set of $\left\{\Delta J, \Delta K_{a}, \Delta K_{c}\right\}$, rates are approximatively constant, that is, do not strongly depend on $J, K_{a}$ and $K_{c}$. The

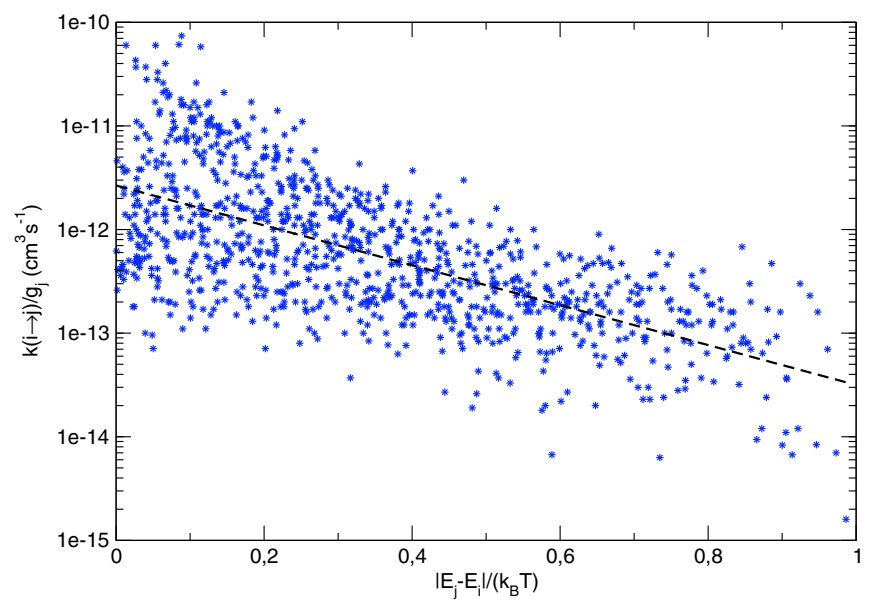

Fig. 1. Downward rotational rate coefficients of Faure et al. (2007) (divided by the degeneracy in the final rotational state) for ortho- $\mathrm{H}_{2} \mathrm{O}$ colliding with para- $\mathrm{H}_{2}$ as a function of the energy gap. The kinetic temperature is $2000 \mathrm{~K}$. The dashed line corresponds to a linear least squares fit of the logarithm of the data.

sum of downward rates out of any upper state and restricted to transitions that do not obey Eq. (3):

$k^{\text {down }}(i ; T)=\sum_{j<i, j \in P} k(i \rightarrow j ; T)$,

is also approximatively constant (in Eq. (4), the $P$ set includes only transitions with $|\Delta J|>2$ or $\left|\Delta K_{a}\right|>2$ or $\left|\Delta K_{c}\right|>2$ ). We can therefore classify collision-induced rotational transitions into two categories: high propensity transitions that do obey Eq. (3) and low propensity transitions that do not. For the former, we define for each set of $\left\{\Delta J, \Delta K_{a}, \Delta K_{c}\right\}$ an average high propensity downward rate:

$k^{\mathrm{hp}}(i \rightarrow j ; T)=k_{\Delta J, \Delta K_{a}, \Delta K_{c}}(T)$,

computed using the corresponding rates within the lowest 990 transitions. For the latter, we compute the average of $k^{\text {down }}(i ; T)$ for the lowest 45 levels, $k^{\text {down }}(T)$, and we define the low propensity downward rates to be:

$k^{\operatorname{lp}}(i \rightarrow j ; T)=\frac{g_{j} k^{\mathrm{down}}(T)}{S(J \alpha ; T)} \exp \left[-\theta_{R}(T)\left|\Delta E_{i j}\right| / k_{B} T\right]$,

where

$S(i ; T)=\sum_{k<i, k \in P} g_{k} \exp \left[-\theta_{R}(T)\left|\Delta E_{i k}\right| / k_{B} T\right]$.

It should be noted that Eq. (6) has similarities with the extrapolation formula used by Neufeld \& Melnick (1987) and Neufeld \& Kaufman (1993).

In Fig. 2, we compare rates calculated from the combination of Eqs. (5) and (6) with the original data of Faure et al. (2007). The high propensity rates are easy to identify because they correspond to "peaks" in the plots (see in particular the lower panel). It can be noticed that Eq. (5) does successfully reproduce the largest peaks. Equation (6) is also found to reproduce the general trend of the low propensity rates, although the lowest rates (those below $\sim 10^{-13} \mathrm{~cm}^{3} \mathrm{~s}^{-1}$ ) are generally overestimated. These low rates are expected to play only a minor role in the radiative transfer equations. As a result, the present method based on the combination of Eqs. (5) and (6) is found to provide a good, quantitative estimate of the highest rotational rates 


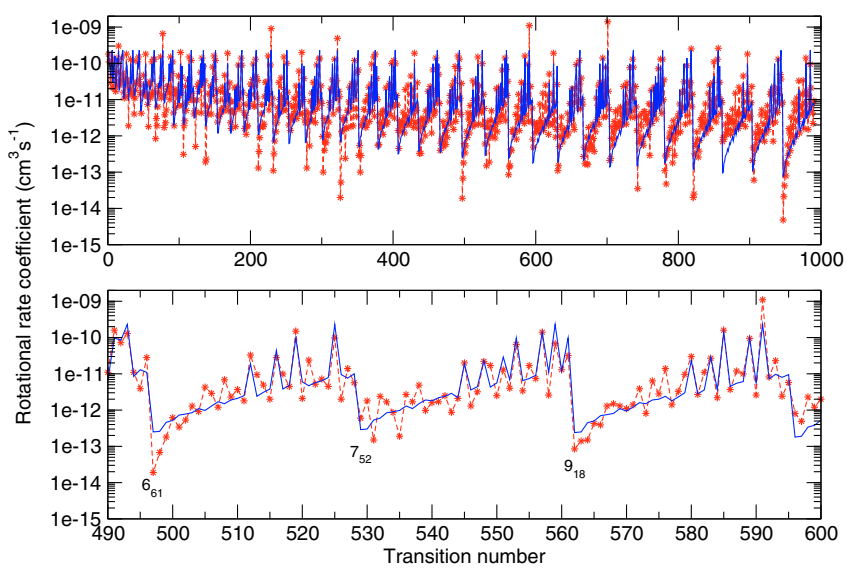

Fig. 2. Downward rotational rate coefficients for ortho- $\mathrm{H}_{2} \mathrm{O}-$ para- $\mathrm{H}_{2}$ at $2000 \mathrm{~K}$ as a function of the transition number (1-990) corresponding to the lowest 45 levels. The original data of Faure et al. (2007) (red stars and dashed line) is compared with our estimate based on the combination of Eqs. (5) and (6) (blue solid line). The lower panel zooms in on the range 490-600, corresponding to transitions involving initial levels $6_{61}, 7_{52}$, and $9_{18}$. See text for details.

with a typical accuracy of factors of $2-3$. Since the original data of Faure et al. (2007) are generally in error by factors of 1-3 (Dubernet et al., private communication), the present data are believed to be accurate within a factor of $\sim 5$ for the highest rates (those above $\sim 10^{-11} \mathrm{~cm}^{3} \mathrm{~s}^{-1}$ ). For lower rates, our extrapolation method is inevitably less accurate and some individual rates may be in error by (at most) one order of magnitude (except for the lowest rates, for which overestimates by 2 orders of magnitude may be found). We emphasize, however, that only comparisons with experimental data will enable the accuracy of the original data of Faure et al. (2007) and, therefore, of the present rates to be assessed reliably. In this context, it should be noted that stateto-state inelastic scattering experiments on $\mathrm{H}_{2} \mathrm{O}-\mathrm{H}_{2}$ are being performed in Nijmegen (ter Meulen, private communication, see e.g. Moise et al. 2007). Detailed comparisons between theory and experiment are in progress and will be published elsewhere.

We also computed downward summed rates (rates from a level summed over all possible downward transitions) for ortho$\mathrm{H}_{2} \mathrm{O}$ colliding with para- $\mathrm{H}_{2}$ using both the QCT data of Faure et al. (2007) and unpublished quantum data of Dubernet et al. (in preparation). Relative differences between the QCT and the quantum summed rates were found to be lower than $50 \%$ for the 10 lowest ortho- $\mathrm{H}_{2} \mathrm{O}$ levels ${ }^{1}$ in the temperature range 200-1500 K, indicating a far higher accuracy for downward summed rates than for individual state-to-state rates. It should be noted that these summed rates are used to estimate critical densities (see Sect. 3.2).

We conclude that the information theory approach, supplemented by both low-lying level data and the knowledge of collisional propensity rules, provides a good framework for extrapolating $\mathrm{H}_{2} \mathrm{O}-\mathrm{H}_{2}$ rotational rates to higher-lying levels. In the present study, the above procedure was applied to all para and ortho rotational transitions involving levels above the lowest 45 and below a threshold energy of $5000 \mathrm{~cm}^{-1}$ with respect to the ground rotational state (corresponding to the 165 th para and 164th ortho level). For temperatures above $2000 \mathrm{~K}$, a simple $T^{1 / 2}$ temperature dependence was assumed for the rates,

\footnotetext{
${ }^{1}$ Quantum data for higher levels are not available when para- $\mathrm{H}_{2}$ is in a rotational state $J_{2}>2$.
}

as suggested by the high temperature results of Faure et al. (2007). Finally, since we are interested in high temperatures $(T \geq 200 \mathrm{~K})$, an $\mathrm{H}_{2}$ ortho-to-para ratio (OPR) of 3:1 was assumed for all temperatures. We note that this choice may be inadequate in some cases, for example in molecular outflows where the OPR has been found to be well below its value at local thermodynamical equilibrium (LTE) (Lefloch et al. 2003; Neufeld et al. 2006). However, the dependence of the $\mathrm{H}_{2} \mathrm{O}$ rates on the $\mathrm{H}_{2}$ OPR has been shown to be modest above $200 \mathrm{~K}$ (Faure et al. 2007).

\subsection{2. $\mathrm{H}_{2} \mathrm{O}$-electron}

Rate coefficients for electron-impact excitation of water were computed by Faure et al. (2004) for rotational de-excitation among the lowest 14 para and 14 ortho rotational levels of $\mathrm{H}_{2} \mathrm{O}$ (corresponding to $J \leq 5$ ) in the temperature range 100-8000 K. This corresponds to a total of 91 para and 91 ortho $\mathrm{H}_{2} \mathrm{O}$ downward rates for each temperature. These calculations were performed by combining the molecular $R$-matrix method with the adiabatic-nuclei-rotation (ANR) approximation. An alternative theory, for which state-to-state cross sections are derived from experimental data, was also reported (Čurík et al. 2006). Both approaches were found to be in good agreement, confirming the accuracy of the $R$-matrix calculations. Faure et al. (2004) also found that $\mathrm{H}_{2} \mathrm{O}$-electron rotational rates are dominated by dipolar transitions:

$\Delta J=0, \pm 1 ; \quad \Delta K_{a}= \pm 1, \pm 3, \ldots ; \quad \Delta K_{c}= \pm 1, \pm 3, \ldots$

They also demonstrated that the accuracy of the Born approximation, which considers only the electron-dipole interaction, increases with the collision energy (see Fig. 2 of Faure et al. 2004). Since we are interested in high temperatures $(T>200 \mathrm{~K})$, rates for dipole-allowed transitions involving levels with $J>5$ were computed using the Born approximation. For dipole-forbidden transitions, Eq. (6) was computed using the original data of Faure et al. (2004). In Fig. 3, we can first observe that the high propensity rates, that is those obeying Eq. (8), are well reproduced by the Born calculation, as expected at such a high temperature (2000 K). Second, Eq. (6) is again found to reproduce the general trend of the low propensity rates, although it overestimates those lower than $\sim 10^{-10} \mathrm{~cm}^{3} \mathrm{~s}^{-1}$. Finally, we note that that the highest rates exceed those for $\mathrm{H}_{2} \mathrm{O}-\mathrm{H}_{2}$ by typically four orders of magnitude (see discussion on cooling rates in Sect. 3.1).

We conclude that the information theory approach supplemented by both low-lying level data and the Born approximation provides a good framework for extrapolating $\mathrm{H}_{2} \mathrm{O}$-electron rotational rates to higher-lying levels. In the present study, the above procedure was applied to all para and ortho- $\mathrm{H}_{2} \mathrm{O}$ levels above the lowest 14 and below a threshold energy of $5000 \mathrm{~cm}^{-1}$, as for $\mathrm{H}_{2} \mathrm{O}-\mathrm{H}_{2}$.

\subsection{Vibrational excitation}

\subsection{1. $\mathrm{H}_{2} \mathrm{O}-\mathrm{H}_{2}$}

Faure et al. (2005a) computed quasi-classical rate coefficients for the relaxation of the first excited bending mode of $\mathrm{H}_{2} \mathrm{O}$ (lying at $1594.7 \mathrm{~cm}^{-1}$ above the ground state) by $\mathrm{H}_{2}$. Their high temperature $(T \geq 1500 \mathrm{~K})$ relaxation rate coefficients were found to be large $\left(>10^{-11} \mathrm{~cm}^{3} \mathrm{~s}^{-1}\right)$ and compatible with the single experimental value at $295 \mathrm{~K}$ (Zittel \& Masturzo 1991), as illustrated in Fig. 4. At lower temperatures ( $T<1500 \mathrm{~K})$, however, the quasi-classical trajectory (QCT) approach was 


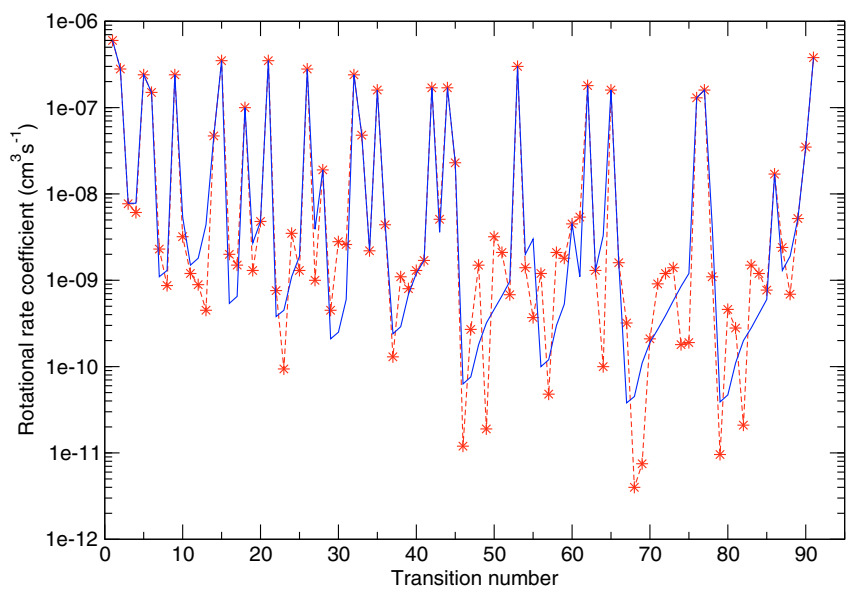

Fig. 3. Downward rate coefficients for electron-impact rotational excitation of ortho- $\mathrm{H}_{2} \mathrm{O}$ at $2000 \mathrm{~K}$ as a function of the transition number (1-91) corresponding to the lowest 14 levels. The original data of Faure et al. (2004) (red stars and dashed line) is compared with our estimate based on a combination of the Born approximation and Eq. (6) (blue solid line). See text for details.

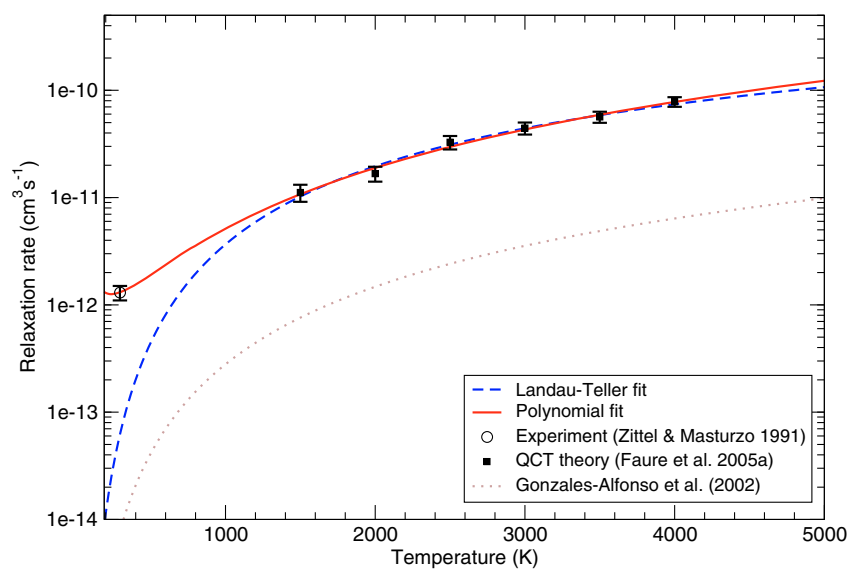

Fig. 4. Rate coefficients for the relaxation of the first excited bending mode of water by $\mathrm{H}_{2}$ as a function of temperature. QCT results are plotted as filled squares (with error bars corresponding to two Monte Carlo standard deviations). The solid line indicates the interpolation of the QCT data and the experimental point using the polynomial form of Eq. (14). This corresponds to our recommended values. The dashed line denotes a $T^{-1 / 3}$ fit, as predicted by the Landau-Teller classical model. Finally, values previously used in the astrophysical literature are indicated by a dotted line (see text for details).

shown to be questionable, due to small number statistics. This problem is, of course, even more severe for state-to-state rovibrational transitions. Quantum mechanics is computationally prohibitive for such a system, particularly because the standard rotational infinite-order-sudden (IOS) approximation is unreliable for molecules with high rotational constants (Faure et al. 2005b, and references therein). As a result, the calculation of state-to-state rate coefficients for the vibrational (de)excitation of $\mathrm{H}_{2} \mathrm{O}$ by $\mathrm{H}_{2}$ appears highly challenging from both a classical and quantum point of view. We develop below an approximate method to estimate rates for state-to-state transitions connecting two vibrational states of water, by considering the lowest 5 vibrational levels denoted by $v=(v 1, v 2, v 3)^{2}$. The first rotational levels of the four lowest excited vibrational levels are

\footnotetext{
${ }^{2}$ In the standard normal mode notation (which is valid for low levels of the stretching excitation), $v=(v 1, v 2, v 3)$ represents vibrational
}

at $1594.7 \mathrm{~cm}^{-1}(010), 3151.6 \mathrm{~cm}^{-1}(020), 3657.1 \mathrm{~cm}^{-1}(100)$, and $3755.9 \mathrm{~cm}^{-1}(001)$ above the ground state (000) (Tennyson et al. 2001).

It was generally assumed in the astronomical literature (see e.g. Chandra \& Karma 2001) that state-to-state ro-vibrational rates are to be proportional to the rates for the corresponding rotational transitions in the ground vibrational state:

$k\left(v, i \rightarrow v^{\prime}, j ; T\right)=P_{v v^{\prime}} k(000, i \rightarrow 000, j ; T)$,

where $\left\{v, v^{\prime}\right\}$ specify respectively the initial and final $\mathrm{H}_{2} \mathrm{O}$ vibrational quantum numbers and $P_{v v^{\prime}}$ is a dimensionless parameter. For the (de)excitation of the bending mode (010) of water, $P_{v v^{\prime}}$ was generally assumed to have a value between 0.01 and 1 (e.g. Gonzalez-Alfonso et al. 1998) and to be independent of temperature.

In their QCT study, Faure et al. (2005b) demonstrated that the final distribution of the water rotational levels after a vibrational relaxation is similar to the final distribution after a pure rotational relaxation: it peaks at the initial $J$ with an exponential decay as a function of the rotational energy gap (see Fig. 3 of Faure et al. 2005b). Exception occurs for low initial $J$, in which case the rotational distribution is broad and peaks at higher $J$. We note that similar results were observed for simpler diatomic systems (see for example the quantum VCC-IOS calculations of Lique \& Spielfiedel 2007 for CS-He). The QCT results of Faure et al. (2005b) therefore suggest that Eq. (9), which corresponds to decoupling rotation and vibration, is qualitatively correct. It should be noted, however, that Eq. (9) completely neglects quasiresonant, vibration-rotation, energy-transfer, which is expected to occur at low energy or for high initial $J$ (see Faure et al. 2005b, and references therein). Furthermore, vibrational transitions for which the initial and final rotational quantum numbers are identical require special consideration: in this case, Eq. (9) does not apply because rotationally elastic rates are typically 1-2 orders of magnitude higher than rotationally inelastic rates. For vibrationally inelastic transitions, this propensity is still marked but it is significantly reduced (Faure et al. 2005b; Lique \& Spielfiedel 2007). Our recommendation for these transitions is therefore to employ the highest rate from the considered initial state.

To define $P_{v v^{\prime}}$, our guiding principle is that of detailed balance, which states that upward and downward ro-vibrational rates must obey the relation:

$k\left(v, i \rightarrow v^{\prime}, j ; T\right)=\frac{g_{j}}{g_{i}} \exp \left[\left(E_{v, i}-E_{v^{\prime}, j} / k_{B} T\right] k\left(v^{\prime}, j \rightarrow v, i ; T\right)\right.$.

By expressing the rotationally summed and averaged vibrational relaxation rate to be:

$k\left(v \rightarrow v^{\prime} ; T\right)=\frac{\sum_{i} g_{i} \exp \left[-E_{v, i} / k_{B} T\right] \sum_{j} k\left(v, i \rightarrow v^{\prime}, j ; T\right)}{\sum_{i} g_{i} \exp \left[-E_{v, i} / k_{B} T\right]}$,

and assuming

$E_{v^{\prime}, i}=E_{v, i}+\Delta E_{v v^{\prime}}$

where $\Delta E_{v v^{\prime}}$ is the vibrational energy gap, we can show that the following definition of $P_{v v^{\prime}}$ :

$P_{v v^{\prime}}(T)=\frac{k\left(v \rightarrow v^{\prime} ; T\right) \sum_{i} g_{i} \exp \left[-E_{v, i} / k_{B} T\right]}{\sum_{i} g_{i} \exp \left[-E_{v, i} / k_{B} T\right] \sum_{j} k(0, i \rightarrow 0, j ; T)}$,

quanta in normal modes $v 1$ (symmetric stretch), $v_{2}$ (bending), and $v_{3}$ (antisymmetric stretch), respectively. 
ensures that detailed balance is automatically fulfiled by Eq. (9). We note that $P_{v v^{\prime}}$ depends on temperature. Obviously Eq. (12) is not rigorously exact (see below), although the main source of inaccuracy is the assumption of complete decoupling between rotation and vibration. We, nevertheless, believe that substituting Eqs. (13) into (9) provides the essential characteristics of the inelastic, state-to-state, vibrational processes involving water.

In Fig. 4, experimental and QCT relaxation rate coefficients for the lowest vibrationally excited mode of water, (010), are plotted as a function of temperature. We added to this figure a previous estimate based on an empirical equation derived from shock-tube measurements of diatomic systems (see e.g., González-Alfonso et al. 2002, and references therein). It can be noticed that this empirical prediction underestimates the experimental rate (at $295 \mathrm{~K}$ ) by more than two orders of magnitude and the QCT rates (at higher temperature) by typically one order of magnitude. The vibrational (de)excitation of water by $\mathrm{H}_{2}$ is therefore far more rapid than anticipated.

QCT and experimental data were interpolated using the polynomial form:

$$
\ln k(T)=\sum_{i=0}^{3} a_{i} T^{-i / 3},
$$

which was found to provide an accurate representation of the rate in the range $200-5000 \mathrm{~K}$, as shown in Fig. 4. The fitting coefficients are the following: $a_{0}=-14.01, a_{1}=-202.1, a_{2}=960.0$, and $a_{3}=-1374$. It should be noticed that a standard $T^{-1 / 3}$ law, as predicted by the Landau-Teller classical model, does also fit the QCT data satisfactory in the high temperature region. Below $1500 \mathrm{~K}$, however, the $T^{-1 / 3}$ dependence does not hold. This finding was observed in many other hydrogen systems as demonstrated by strongly nonlinear Landau-Teller plots (see for example the theoretical work of Dashevskaya et al. (2007) for $\mathrm{H}_{2}+\mathrm{H}_{2}$ ). Furthermore, even negative temperature dependence was observed experimentally close to room temperature for the self-relaxation of $\mathrm{H}_{2} \mathrm{O}$ (Zittel \& Masturzo 1991). It is therefore possible that the rate for vibrational relaxation of $\mathrm{H}_{2} \mathrm{O}$ by $\mathrm{H}_{2}$ increases below $300 \mathrm{~K}$.

For higher vibrational modes of water, the only available data is provided by the measurements of Zittel \& Masturzo (1991) at $295 \mathrm{~K}$. In addition to the (010) bending level, these authors measured rate coefficients for vibrational relaxation of the (100) and (001) stretching level reservoir and the (020) bending overtone. Since the relaxation between the nearly resonant (100) and (001) stretching levels is far more rapid than relaxation from the stretching levels, (100) and (001) were experimentally found to reach equilibrium immediately and to relax as a single reservoir. Rates for relaxation of the stretching level reservoir are therefore the Boltzmann-weighted averages of the rates for the individual (100) and (001) levels. Zittel \& Masturzo (1991) measured total relaxation rate constants and only some information was deduced about the individual, state-to-state vibrational rates. As a result, the following assumptions were made in the present work:

$k(001 \rightarrow 100 ; T)=k(000 \rightarrow 000 ; T)$,

and

$k\left(001 \rightarrow v^{\prime} ; T\right)=k\left(100 \rightarrow v^{\prime} ; T\right)=k\left(v_{1,3} \rightarrow v^{\prime} ; T\right)$,

where $v_{1,3}$ designates the stretching level reservoir. We also assumed that all vibrationally elastic rates, that is rates for pure rotational transitions within a given vibrational level, are equal to the rotational rates within the ground vibrational state $(000)$.
Table 1. Rate constants for vibrational relaxation of $\mathrm{H}_{2} \mathrm{O}$ by $\mathrm{H}_{2}$ at $295 \mathrm{~K}$ derived from the experiment of Zittel \& Masturzo (1991). Powers of 10 are given in parentheses.

\begin{tabular}{ccc}
\hline \hline Initial level & Final level & $k\left(\mathrm{~cm}^{3} \mathrm{~s}^{-1}\right)$ \\
\hline 010 & 000 & $1.30(-12)$ \\
020 & 010 & $2.30(-12)$ \\
020 & 000 & $0.60(-12)$ \\
100 & 020 & $0.64(-12)$ \\
100 & 010 & $0.08(-12)$ \\
100 & 000 & $0.08(-12)$ \\
001 & 020 & $0.64(-12)$ \\
001 & 010 & $0.08(-12)$ \\
001 & 000 & $0.08(-12)$ \\
\hline
\end{tabular}

This assumption was tested by QCT calculations on the lowest (010) mode and found to be accurate within typically two Monte Carlo standard deviations.

From Tables 1, 2 of Zittel \& Masturzo (1991), vibrational relaxation rates at $295 \mathrm{~K}$ among the lowest 5 vibrational levels were determined and are summarized in Table 1 . We note that the parameter $Z_{\mathrm{H}_{2}}=k_{31, \mathrm{H}_{2}}^{\prime} / k_{32, \mathrm{H}_{2}}$ (see Eqs. (1)-(3) of Zittel \& Masturzo 1991) is taken to be 0.15 . We also assumed that the rate for relaxation from the $v_{1,3}$ reservoir to the ground state equals the rate for relaxation to the (010) level (see discussion in Zittel \& Masturzo 1991). Finally, vibrational excitation of $\mathrm{H}_{2}$ was neglected, in agreement with experimental results (see Table 2 of Zittel \& Masturzo 1991).

The rate constants presented in Table 1 were extrapolated from 200 to $5000 \mathrm{~K}$, assuming the same temperature dependence as the rate for the $(010) \rightarrow(000)$ transition (see Eq. (14)). This hypothesis should be reasonable, especially at high temperatures $(T \gtrsim 1000 \mathrm{~K})$ where all rates are expected to follow a Landau-Teller law. The coefficients $P_{v v^{\prime}}(T)$, represented by Eq. (13), were then computed for each vibrational transition using the above defined rates and the experimental level energies taken from Tennyson et al. (2001). The coefficients $P_{v v^{\prime}}(T)$ were found to be similar for ortho and para- $\mathrm{H}_{2} \mathrm{O}$, typically to within $5 \%$, and to have values between $10^{-4}$ and $10^{-1}$, as illustrated in Fig. 5. The coefficients $P_{v v^{\prime}}(T)$ were found to vary significantly (over an order of magnitude) in the temperature range $200-5000 \mathrm{~K}$, with a minimum at $400 \mathrm{~K}$. This minimum reflects the competition in Eq. (13) between the temperature dependences of $k\left(v \rightarrow v^{\prime} ; T\right)$ and the sums in the numerator and denominator.

Finally, we note that Eq. (13) can be applied to both excitation and de-excitation transitions. We recommend using the equation for de-excitation (relaxation) transitions for which rates have been measured. However, we computed $P_{v v^{\prime}}(T)$ as a test for excitation transitions. The resulting state-to-state rate constants were found to satisfy detailed balance to within typically $20 \%$. This indicates that Eq. (12) does not introduce major inacurracies.

In the present study, the above procedure was applied to all para and ortho ro-vibrational de-excitation transitions involving levels below a threshold energy of $5000 \mathrm{~cm}^{-1}$ with respect to the ground state. This corresponds to 108 para and 107 ortho rotational levels within (010), 56 para and 55 ortho levels within (020), 44 para and 44 ortho levels within (100) and, finally, 41 para and 40 ortho levels within (020). This results in a total of 824 levels, including those in the ground vibrational state (000). The complete set of de-excitation rates among all levels between 200 and $5000 \mathrm{~K}$ are provided as online material 


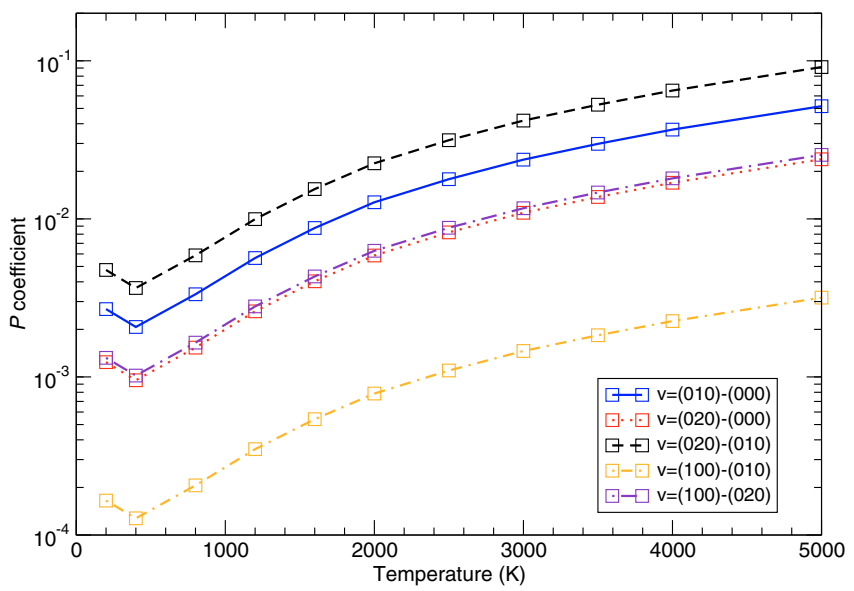

Fig. 5. Temperature dependence of the $P_{v v^{\prime}}(T)$ coefficients for different vibrational transitions, as defined in Eq. (13), for (ortho-) $\mathrm{H}_{2} \mathrm{O}-\mathrm{H}_{2}$ collisions. Note that only five over the nine transitions are plotted for clarity in the figure.

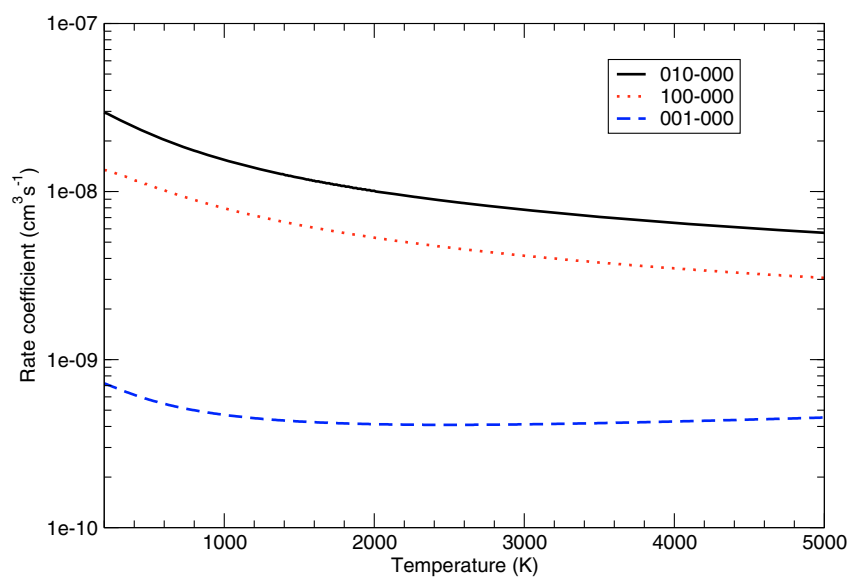

Fig. 6. Rate coefficients for the relaxation of the three normal modes of water by electron-impact, calculated from the cross sections of Nishimura \& Gianturco (2004).

(Tables A.1, A.2). Excitation rates can be obtained from the detailed balance relation, Eq. (10). Tables A.1, A.2 will be also made available in the BASECOL database ${ }^{3}$ and at the $\mathrm{CDS}^{4}$.

\subsection{2. $\mathrm{H}_{2} \mathrm{O}$-electron}

Nishimura \& Gianturco (2004) computed quantum vibrationally inelastic cross-sections for the water molecule colliding with low-energy electrons. Results were obtained to low energies for the three normal modes of $\mathrm{H}_{2} \mathrm{O}(010,100$, and 001) and good agreement with existing experiments was found. From their cross sections (tabulated in Table 3 of their paper), we computed thermal relaxation rate coefficients for the temperature range $200-5000 \mathrm{~K}$. These rates are plotted in Fig. 6. In contrast to $\mathrm{H}_{2} \mathrm{O}-\mathrm{H}_{2}$ relaxation rates, we note that electron-impact relaxation rates decrease with increasing temperature. This reflects the energy dependence of the excitation cross-sections, which show strong peaks close to thresholds, especially the $v_{1}$ and $v_{2}$ modes (see Nishimura \& Gianturco 2004). As a result, excitation rates decline slowly to zero at low temperature. This

\footnotetext{
3 http://wwW.obspm.fr/basecol/

${ }^{4}$ http://cdsweb.u-strasbg.fr/cgi-bin/qcat?J/A+A/492/ 257
}

Table 2. Fitting coefficients $a_{i}(i=0$ to 3 ) of the polynomial fit, Eq. (14), to the vibrational relaxation rates of $\mathrm{H}_{2} \mathrm{O}$ by electron-impact.

\begin{tabular}{cccccc}
\hline \hline Initial level & Final level & $a_{0}$ & $a_{1}$ & $a_{2}$ & $a_{3}$ \\
\hline 010 & 000 & -21.54 & 57.26 & -254.2 & 368.0 \\
100 & 000 & -22.17 & 58.31 & -271.4 & 398.8 \\
001 & 000 & -19.57 & -64.22 & 629.4 & -1794 \\
\hline
\end{tabular}

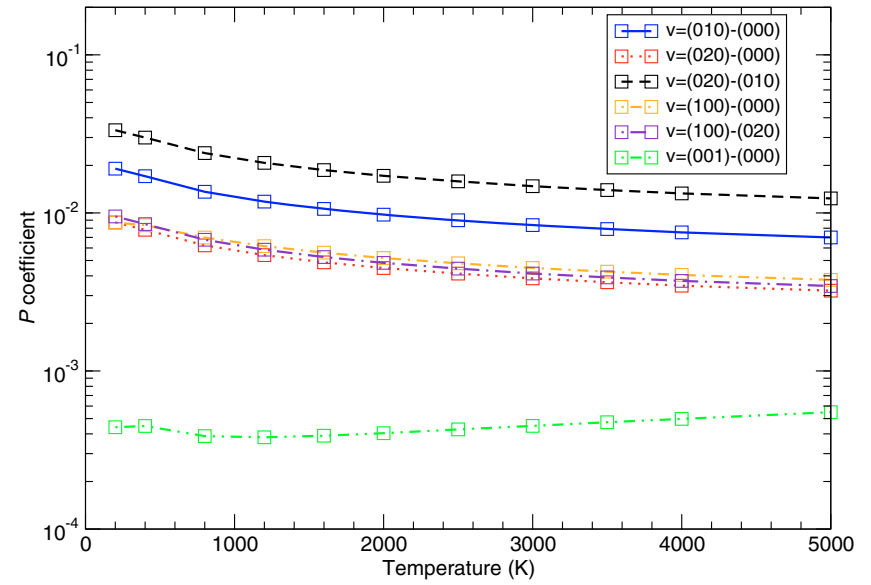

Fig. 7. Temperature dependence of the $P_{v v^{\prime}}(T)$ coefficients for different vibrational transitions, as defined in Eq. (13), for (ortho-) $\mathrm{H}_{2} \mathrm{O}$-electron collisions. Note that only six over the nine transitions are plotted for clarity in the figure.

behaviour is generally attributed to the dominant long-range dipole potential terms at low impact energies. Furthermore, the fact that the $v_{1}$ and $v_{2}$ modes have significantly higher rates than $v_{3}$ is due to much higher polarizability and quadrupole gradients (Nishimura \& Gianturco 2004). Finally, as in the case of rotational excitation, we note that these rates are several orders of magnitude higher than those for $\mathrm{H}_{2} \mathrm{O}-\mathrm{H}_{2}$ (see discussion on cooling rates in Sect. 3.1).

The relaxation rates plotted in Fig. 6 were interpolated using the polynomial form of Eq. (14). The fitting coefficients are given in Table 2.

To our knowledge, neither theoretical nor experimental data are available in the literature for other transitions. This is true in particular for those involving the $v_{2}=2$ mode for which we have employed the polynomial fit of the $(010)-(000)$ transition (Table 2) and applied the corresponding ratios observed by Zittel \& Masturzo (1991) for $\mathrm{H}_{2} \mathrm{O}-\mathrm{H}_{2}$ rates, deduced from Table 1. Rates for transitions from (100) and (001) to (010) were also assumed to be identical to those for $(000)$, as for $\mathrm{H}_{2} \mathrm{O}-\mathrm{H}_{2}$. In other words, for transitions not included in Table 2, we assumed the same propensity rules as in the case of $\mathrm{H}_{2} \mathrm{O}-\mathrm{H}_{2}$ (see Table 1). Equation (15) was also assumed.

The corresponding coefficients $P_{v v^{\prime}}(T)$ have values between a few $10^{-4}$ and a few $10^{-2}$, as illustrated in Fig. 7. In contrast to the $\mathrm{H}_{2} \mathrm{O}-\mathrm{H}_{2}$ case, however, the coefficients $P_{v v^{\prime}}(T)$ are found to decrease with increasing temperature and depend only weakly on the temperature (in the range 200-5000 K). This of course reflects the temperature dependence of the rates, as plotted in Fig. 6.

As for $\mathrm{H}_{2} \mathrm{O}-\mathrm{H}_{2}$, Eq. (13) was applied to all para and ortho ro-vibrational de-excitation transitions involving levels below a threshold energy of $5000 \mathrm{~cm}^{-1}$ with respect to the ground state. The complete set of de-excitation rates for all levels between 200 and $5000 \mathrm{~K}$ are provided in online material at the CDS (Tables A.3, A.4). Excitation rates can be obtained from 
the detailed balance relation in Eq. (10). Tables A.3, A.4 will also be made available in the BASECOL database ${ }^{5}$ and at the $\mathrm{CDS}^{6}$.

\section{Astrophysical implications}

Detailed analysis of the implications of our collisional rates requires radiative transfer calculations, which will be presented in a forthcoming paper (Josselin \& Faure, in preparation). We restrict ourselves to basic considerations, by comparing cooling rates and critical densities with those derived from previously assumed hypotheses, such as a constant (independent of temperature) proportionality between rovibrational and rotational rates.

\subsection{Cooling rates}

To illustrate the overall difference between the present new rates and those previously employed in the literature, we calculated the cooling rate in the optically thin low-density limit:

$L_{0}(T)=\sum_{i} k_{0 i} E_{i}$

For both rotational cooling and vibrational cooling, $L_{0}(T)$ was computed previously by Neufeld \& Kaufman (1993). The water ortho-to-para ratio is assumed to be $3: 1$. We calculated Eq. (17) for both $\mathrm{H}_{2} \mathrm{O}-\mathrm{H}_{2}$ and $\mathrm{H}_{2} \mathrm{O}$-electron using the rates described in Sects. 2.1 and 2.2, that is including all levels below $5000 \mathrm{~cm}^{-1}$. In Figs. 8 and 9, comparisons between the present results and those of Neufeld \& Kaufman (1993) are presented. It can be noticed that the new $\mathrm{H}_{2} \mathrm{O}-\mathrm{H}_{2}$ rotational rates increase the cooling rate at low temperature, as expected, but decrease the rate at high temperature with respect to the values of Neufeld \& Kaufman (1993). This reflects the different extrapolation methods used here and in the work of Neufeld \& Kaufman (1993). We also observe that the $\mathrm{H}_{2} \mathrm{O}$-electron rotational cooling rate is higher than the $\mathrm{H}_{2} \mathrm{O}-\mathrm{H}_{2}$ cooling rate by several orders of magnitude, as expected (see Sect. 2.1.2). We note, however, that the electron fraction, $x(e)=[e] /[H]$, is generally in the range $\left[10^{-9}-10^{-3}\right]$, so that the contribution of electrons is significant only in the harsher environments $\left(x(e)>10^{-4}\right)$, e.g. photon-dominated regions (PDRs) and $\mathrm{X}$-ray dominated regions (XDRs).

In Fig. 9, it can be noticed that the new $\mathrm{H}_{2} \mathrm{O}-\mathrm{H}_{2}$ vibrational rates increase the cooling rate by one to three orders of magnitude with respect to the value of Neufeld \& Kaufman (1993). The vibrational cooling rates are still lower than the rotational rates except at $T>3000 \mathrm{~K}$ where they are comparable. We note, however, that Neufeld \& Kaufman (1993) demonstrated that cooling due to vibrational transitions of $\mathrm{H}_{2} \mathrm{O}$ may dominate over rotational transitions at high temperature and $\mathrm{H}_{2}$ density when opacity and non-LTE effects are taken into account (see Fig. 6 of their article). We also note that the vibrational cooling rate is essentially dominated by the lowest vibrational mode $(010)$ at these temperatures. Finally, the $\mathrm{H}_{2} \mathrm{O}$-electron vibrational cooling rate exceeds the $\mathrm{H}_{2} \mathrm{O}-\mathrm{H}_{2}$ rate by two to four orders of magnitude.

\subsection{Critical densities}

The concept of a critical density, i.e. that above which collisions dominate over radiative processes in populating energy levels

\footnotetext{
5 http://wwW.obspm.fr/basecol/

${ }^{6}$ http://cdsweb.u-strasbg.fr/
}

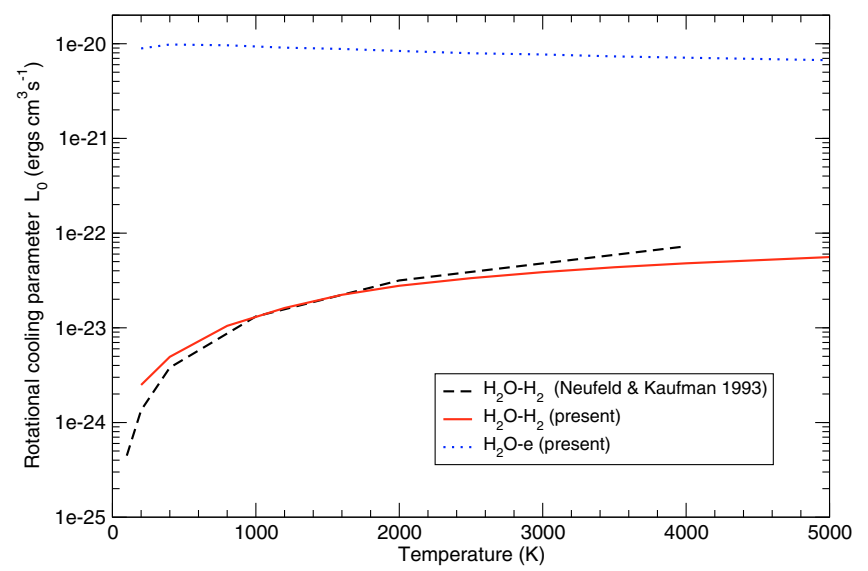

Fig. 8. Rotational cooling rates in the optically thin low-density limit, Eq. (17), as a function of temperature. The present $\mathrm{H}_{2} \mathrm{O}-\mathrm{H}_{2}$ and $\mathrm{H}_{2} \mathrm{O}$-electron rates are compared to the $\mathrm{H}_{2} \mathrm{O}-\mathrm{H}_{2}$ results of Neufeld \& Kaufman (1993).

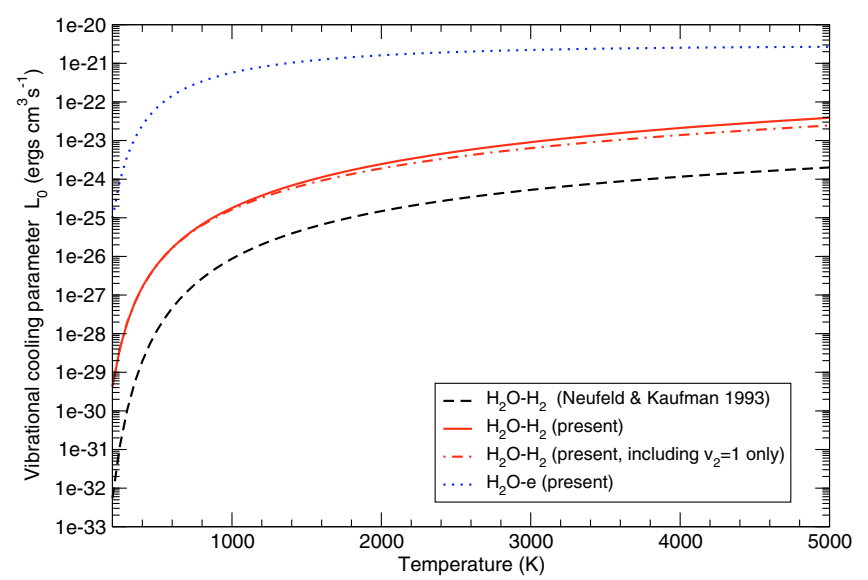

Fig. 9. Vibrational cooling rates in the optically thin low-density limit, Eq. (17), as a function of temperature. The present $\mathrm{H}_{2} \mathrm{O}-\mathrm{H}_{2}$ and $\mathrm{H}_{2} \mathrm{O}$-electron rates are compared with the $\mathrm{H}_{2} \mathrm{O}-\mathrm{H}_{2}$ results of Neufeld \& Kaufman (1993). Present results including only the lowest $v_{2}=1$ level are also plotted.

and ensure local thermodynamic equilibrium (LTE) conditions, is poorly defined in the case of a multi-level system. For a 2-level system, we have: $n_{\text {crit }}=A_{\mathrm{ul}} / k_{\mathrm{ul}}$, where $A_{\mathrm{ul}}$ denotes the Einstein A-coefficient and $k_{\mathrm{ul}}$ the collisional rate coefficient, for the transition from the upper (u) to the lower (l) level. In the case of a multi-level, these coefficients have to be summed over all possible downward transitions. For radiative rates, we use the computed line list published by Barber et al. (2006).

As an illustration, we consider the $(010) 6_{61}-7_{52}$ water line, observed for the first time by Menten et al. (2006) towards the red supergiant star (RSG) VY CMa. The $\mathrm{H}_{2}$ critical density for the $6_{61}$ level as a function of temperature is shown in Fig. 10, with the location of typical conditions for a RSG atmosphere (MARCS spherical model, with $T_{\text {eff }}=3600 \mathrm{~K}, M=15 M_{\odot}$, $\log g=0.0 ;$ Gustafsson et al. 2008), the circumstellar conditions for which this submillimetre line may be formed $(T=1000 \mathrm{~K}$, $n_{\mathrm{H}_{2}}=2 \times 10^{9} \mathrm{~cm}^{-3}$ ), and the $\mathrm{H}_{2}$ critical density derived by Menten et al. (2006): $n_{\text {crit }}\left(6_{61}\right)=5 \times 10^{12} \mathrm{~cm}^{-3}$. These authors assumed that rate coefficients for transitions from the ground to the $v_{2}=1$ state are identical to those for transitions within the ground state with identical quantum numbers multiplied by a constant factor of 0.02 . This is about two orders of magnitude 


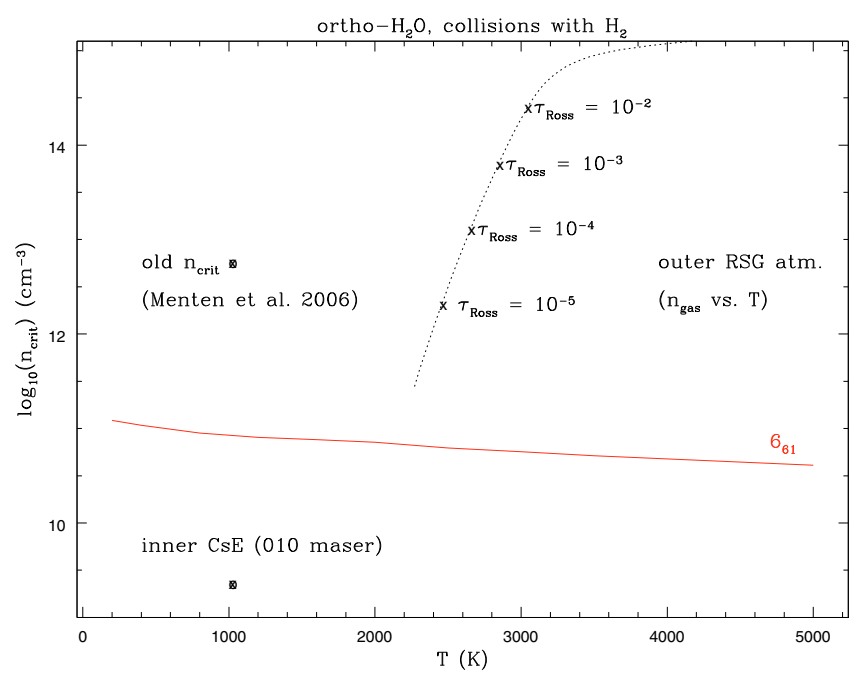

Fig. 10. Critical density for $\mathrm{H}_{2}$ collisions as a function of temperature for the (010) $6_{61}$ level, compared with typical conditions for a RSG atmosphere and circumstellar envelope (see text for details).

larger than the critical density we derive from our collisional rates, mainly because Menten et al. (2006) did not consider vibrationally elastic (i.e. pure rotational) rates within (010). Our new value (about $8 \times 10^{11} \mathrm{~cm}^{-3}$ at $1000 \mathrm{~K}$ ) still implies that collisions with $\mathrm{H}_{2}$ molecules cannot thermalize the observed lines, but their contribution cannot be neglected. In other words, purely radiative excitation now appears questionable for this line (and the other submillimetre maser lines). Furthermore, the gas density rises above the value of the critical density in the transition region between the outer stellar atmosphere and the circumstellar region where the maser lines are supposed to be formed. A detailed, multi-zone, radiative-transfer model is therefore essential to interpret these observations. We finally note that the maser lines are believed to be collisionally-pumped (Humphreys 2007, and references therein). Adopting our new collisional rates, we propose to test the efficiency of $v_{2}=1$ maser pumping with radiative transfer calculations (Josselin \& Faure, in preparation).

\section{Conclusions}

In this paper, we have presented new collisional rate coefficients for the (de)excitation of the lowest 824 ro-vibrational levels of water by both $\mathrm{H}_{2}$ and electrons in warm and hot molecular gas $(200<T<5000 \mathrm{~K})$. Pure rotational rates have been obtained by extrapolating the data of Faure et al. (2004) and Faure et al. (2007) using a new method partly based on the information (phase space) theory of Levine and co-workers. Ro-vibrational rates have been obtained using vibrational relaxation data available in the literature and by assuming complete decoupling between rotation and vibration. Since the published data that we have employed are believed to be accurate to within a factor of 3 , our extrapolated data should be accurate to within a factor of $\sim 5$ for the highest rates (those above $\sim 10^{-11} \mathrm{~cm}^{3} \mathrm{~s}^{-1}$ ). Lower rates, including the ro-vibrational ones, should be generally accurate to within an order of magnitude. Despite these modest accuracies, we note that summed rates (see Sect. 2.1.1) and cooling rates (see Sect. 3.1) have a higher precision and the present data should be adequate for estimating density and temperature conditions from emission spectra. The prediction of physical conditions required to produce non-LTE effects should also be reasonable. We have therefore shown that $\mathrm{H}_{2} \mathrm{O} v_{2}=1$ lines observed in RSG stars are expected to be at least partly excited by collisions. Forthcoming radiative transfer calculations will investigate this point in more detail (Josselin \& Faure, in preparation). Finally, we would like to emphasize that the present study provides the most extensive collisional data to date for an asymmetric rotor.

Acknowledgements. E.J. thanks for their hospitality the Uppsala observatory where part of this work was initiated. This research was supported by the CNRS national program "Physique et Chimie du Milieu Interstellaire", by the FP6 Research Training Network "Molecular Universe" (contract number MRTN-CT-2004-512302), and by the French National Research Agency (ANR) through program number ANR-06-BLAN-0105.

\section{References}

Barber, R. J., Tennyson, J., Harris, G. J., \& Tolchenov, R. N. 2006, MNRAS, 368,1087

Barnes, P. W., Sims, I. R., \& Smith, I. W. M. 2004, J. Chem. Phys., 120, 5592

Chandra, S., \& Sharma, A. K. 2001, A\&A, 376, 356

Čurík, R., Ziesel, J. P., Jones, N. C., Field, T. A., \& Field, D. 2006, Phys. Rev. Lett., 97, 123202

Dashevskaya, E. I., Litvin I., Nikitin, E. E., \& Troe, J. 2007, J. Chem. Phys., 127, 114317

Dubernet, M.-L., Daniel, F., Grosjean, A., et al. 2006, A\&A, 460, 323

Faure, A., \& Wiesenfeld, L. 2004, J. Chem. Phys., 121, 6771

Faure, A., Gorfinkiel, J. D., \& Tennyson, J. 2004, MNRAS, 347, 323

Faure, A., Valiron, P., Wernli, M., et al. 2005a, J. Chem. Phys., 122, 1102

Faure, A., Wiesenfeld, L., Wernli, M., \& Valiron, P. 2005b, J. Chem. Phys., 123, 4309

Faure, A., Wiesenfeld, L., Wernli, M., \& Valiron, P. 2006, J. Chem. Phys., 124, 214310

Faure, A., Crimier, N., Ceccarelli, C., et al. 2007, A\&A, 472, 1029

Gonzalez-Alfonso, E., Cernicharo, J., van Dishoeck, E. F., Wright, C. M., \& Heras, A. 1998, ApJ, 502, L169

González-Alfonso, E., Wright, C. M., Cernicharo, J., et al. 2002, A\&A, 386, 1074

Green, S. 1979, Chem. Phys., 40, 1

Green, S., Maluendes, S., \& McLean, A. D. 1993, ApJS, 85, 181

Gustafsson, B., Edvardsson, B., Eriksson, K., et al. 2008, A\&A, 486, 951

Humphreys, E. M. L. 2007, IAUS, 242, 471

Lefloch, B., Cernicharo, J., Cabrit, S., et al. 2003, ApJ, 590, L41

Levine, R. D. 1978, Ann. Rev. Phys. Chem., 29, 59

Lique, F., \& Spielfiedel, A. 2007, A\&A, 462, 1179

Menten, K. M., Philipp, S. D., Güsten, R., et al. 2006, A\&A, 454, L107

Moise, A., Parker, D. H., \& Ter Meulen, J. J. 2007, J. Chem. Phys., 126, 124302

Neufeld, D. A., \& Melnick, G. J. 1987, ApJ, 322, 266

Neufeld, D. A., \& Kaufman, M. J. 1993, ApJ, 418, 263

Neufeld, D. A., Melnick, G. J., Sonnentrucker, P., et al. 2006, ApJ, 649, 816

Nishimura, T., \& Gianturco, F. A. 2004, Europhys. Lett., 65, 179

Otto, F., Gatti, F., \& Meyer, H.-D. 2008, J. Chem. Phys., 128, 064305

Phillips, T. R., Maluendes, S., \& Green, S. 1996, ApJS, 107, 467

Procaccia, I., \& Levine, R. D. 1976, Physica A, 82, 623

Tennyson, J., Zobov, N. F., Williamson, R., Polyansky, O. L., \& Bernath, P. F. 2001, J. Phys. Chem. Ref. Data, 30, 735

Zittel, P. F., \& Masturzo, D. E. 1988, J. Chem. Phys., 90, 977

Zittel, P. F., \& Masturzo, D. E. 1991, J. Chem. Phys., 95, 8005 